DOI: $10.51480 / 1899-5101.14 .2(29) .4$

\title{
Exploring Visual Culture of COVID-19 Memes: Russian and Chinese Perspectives
}

\author{
Olga V. Smirnova \\ ORCID: 0000-0003-1349-4241 \\ Lomonosov Moscow State University, Moscow,
}

Russia

Alexandre P. Lobodanov

(iD) ORCID: 0000-0003-1113-9297

Lomonosov Moscow State University, Moscow,

Russia

Galina V. Denissova

(D) ORCID: 0000-0002-7719-8380

Lomonosov Moscow State University, Moscow,

\author{
Anna A. Gladkova \\ ORCID: 0000-0001-7102-6425 \\ Lomonosov Moscow State University, Moscow, \\ Russia
}

Olga V. Sapunova

ORCID: 0000-0002-2179-6592

Lomonosov Moscow State University, Moscow,

Russia

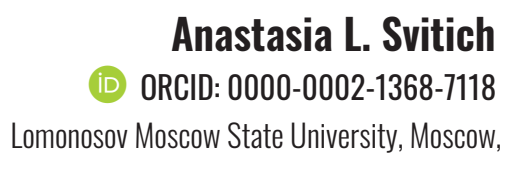

Russia

\begin{abstract}
The article explores COVID-19 related Internet memes and treats them as samples of creolized text in a study of the way ethnic and cultural peculiarities of memes are perceived by representatives of two radically different cultural paradigms: the Russians and Chinese. A survey is used as a method of the investigation. Russian and Chinese students, and visual arts experts evaluated Russian and Chinese COVID-19 memes according to several criteria concerning their content, verbal constituent element and visual characteristics. The study concluded that the social and culture-specific components mostly contribute to forming the opinion of the meme and its appreciation. Awareness of both the cultural background and the current social and nation-specific situation is required to decode a full amount of information contained in a meme. Equally important is the visual component in understanding the meme. Thus, the study contributes not only to studying event-specific memes but also broadens the scope of research on memes as a sample of visual culture.
\end{abstract}

KEYWORDS: Chinese, COVID-19; cultural paradigm; digital memes; visual language; Russian. 


\section{INTRODUCTION}

Information in today's media sphere is transmitted through a variety of verbal, visual, and auditory channels, using numerous codes, such as cultural, ideological, linguistic, semantic, stylistic, graphics, etc. In this context, the phenomenon of the Internet meme, which promptly responds to a newsworthy event and has a viral nature, reflects a wide variety of opportunities for multichannel communication (Blackmore, 1999; Brodie, 1996; Castaño, 2013; Chaoqun \& Ziran, 2007; Shomova, 2018; Wiggins \& Bowers, 2015). At the same time, the Internet meme carries verbal and visual codes of various etymologies and offers a unique opportunity to study the ethnocultural features of the contemporary media discourse.

\section{MEME AS A PART OF VISUAL CULTURE}

The study fits in a broader theoretical paradigm of culture studies, along with visual culture and visual design studies. Following Kress and van Leeuwen (2020), we examine the ways in which images communicate meaning, keeping in mind that "visual language is not transparent and universally understood, but culturally specific" (Kress \& van Leeuwen, 2020, p. 3). By comparing Internet memes in this study as examples of mediated images (Aiello \& Parry, 2019), we focus on aspects of culture that are deemed to be represented in visual form. As earlier discussed by Shifman (2014a, 2014b), we believe that memes may serve as valuable keys for understanding broader dimensions of digital culture. Today, Internet memes represent a new genre of online communication, and an understanding of their production, dissemination, and implications in the real world enables an improved ability to navigate digital culture (Wiggins, 2019). In this paper, we show that the perception of memes as elements of visual communication differs between the two groups: Russians and Chinese. We argue that cultural specifics can affect the way people perceive and interpret the rapidly changing digitalized reality around them.

Here we also refer to the 'meme culture' that in recent years has attracted extensive exploration. In this vein, Iloh $(2021$, p. 3) positions memes as "units that reflect specific contexts and meanings discernable to some and less discernible to others". Some people know, while others may not, the cultural meaning contained and reflected in memes. The inquiry into the cultural information contained in memes has the potential to explicate the ideas, values, repertoires, practices, and conditions that comprise culture (Iloh, 2021, p. 3). Aiello and Parry (2019) show that visual images can be central to understanding culture and its representations, along with peoples' identities. Their study specifically explores 'the role played by visual images in the shaping and maintaining of identities' (Aiello 
\& Parry, 2019, p. 3). We expect this paper therefore to contribute to a broader academic discussion about the relationship between visual images, cultures and identities by exploring and in a way juxtaposing the perception of these two quite diverse ethnic and cultural groups - the Russians and Chinese.

Lastly, we rely in this study on current studies on data visualization, another research area that scholars have in recent years deeply explored. The latest book by Manovich (2020) draws linkages between data visualization, digital culture studies, data science and media studies and further develops concepts of 'cultural software' and 'information aesthetics' suggested in previous works (Manovich, 2017). Large scale projects by Manovich, as well the studies conducted on internet platforms such as Selfiecity, Phototrails and Visual Earth, show that a boundary between science and art is becoming increasingly blurred. Today, there is a clearly a need for new terms, methods and approaches for conceptualizing our rapidly changing digital media landscape, where new languages, forms and representations are constantly emerging and evolving. The study of Internet memes with their growing popularity and role in current visual communication process therefore fits this niche perfectly.

The present research applies the Cultural Discourse Studies (CDS) theoretical paradigm to Internet memes and treats them as samples of using visual culture. We follow Shi-xu $(2016$, p. 3) to argue that nowadays human discourse is being re-conceptualized as a multi-faceted but integrated communicative event, which manifests social interaction using linguistic and other symbolic means and mediums in historical and cultural relations. The focus of our research is the interrelationship between linguistic means (oral or written) and the contextual meaning implicitly encoded in acts of human communication illustrated by comparing and confronting groups of Russians and the Chinese. Though being one of crucial issues of CDS studies, the topic has remained insufficiently considered in academic literature, especially as regards non-Western contexts (Batibo, 2009), and thus it deserves extensive investigation.

The contrast of 'meaning-in-language vs meaning-beyond-language' introduced by Shi-xu (2009, p. 35) describes the way cultural differences contribute to and somewhat shape differences which take place in historical and cultural contexts. Therefore, the aspect to be investigated is a close connection between linguistic means and visual culture elements on the one hand and their context on the other, not a series of isolated images and representations. Cross-cultural studies based on several national contexts (e.g., Russian and Chinese) are considered to be one of the most demonstrative approaches to discuss the role context plays in language, discourse and communication studies. They are also a way to represent culture through various forms and genres of spoken and written texts (including memes, which are a new form of communication). 
However, when it comes to the interplay between textual and visual forms and contexts, another major aspect needs to be emphasized. Cultural and CDS analyses require adopting a less West-centric approach, since 'Eastern wisdoms in understanding the universe can also be mobilized for the paradigmatic reconstruction, for identity, creativity and authenticity' (Shi-xu, 2009, p. 38). Therefore, having selected Russian and Chinese contexts for the current research we intended to shift academic focus to Eastern paradigms, including 'Emerging States' among others, as they are currently understudied (Vartanova \& Gladkova, 2020; Vartanova et al., 2021).).

A visual language is primarily considered by researchers as a complex semiotic structure; the components of this structure interact with each other depending on their functions and position in the general hierarchy of the system of signs. The visual language may be defined as a code system of signs which acquire the status of non-linear visual images in the process of communication, information and knowledge transfer, and other functions. The key function of the components of the visual language is to ensure correct perception of signs and convey the whole meaning of visual messages.

The visual language as a special system of signs has a dual nature: it is a combination of graphic and expressive principles driven by unique sign references. Implications are realized through both general principles of sign formation (formation of forms, composition, rhythm, cadence, and rhythmometry dynamics) and specific principles, such as color, light and shade relations. Implications are usually associated with reconstruction or transformation of the original image, i.e., about the reproduction, interpretation, compilation, and imitation of original signs (Lobodanov, 2013, pp. 135-178).

The impact of implications on sensitivities in the social and cultural perception depends on their producer and the method of sign exchange. The audience may have no special sign formation skills and therefore no sign behavior. In this case, sign exchange is unidirectional: it is directed from the producer of signs to the audience but not the other way around. Moreover, the property of "imagery" in the content of signs is not supplemented by mutual logical or conceptual interpretability, as is the case with signs of the verbal language. For this reason, sign communication in the visual language system is carried out with other characteristics of individual perception than in verbal communication. Here, the level of understanding is crucially dependent on worldview contexts.

In terms of linguistics, the meme is a text containing both verbal and visual components (Sorokin \& Tarasov, 1990, p. 180). Dawkins suggests that a meme better captures the linkage between culture and memory (Dawkins, 1989, p. 192). 
However, as Wiggins suggests

enthymeme better captures the essence of internet memes as a digital phenomenon marked not by imitation but by the capacity to propose or counter a discursive argument through visual and often also verbal interplay; the emphasis here is on those internet memes which inhere a critical component of society, politics, etc. Enthymeme conceptually designates the fundamental differences between meme and internet meme from an orthographic as well as an etymological standpoint (Wiggins, 2019, pp. 16-17).

The visual component of the meme involves illustrative (photo, drawn picture, infographics, animated cartoon image, reproduction, collage) and graphic (using font or decoration) design. Therefore, a meme is a complex convergence of linguistic, extralinguistic, and precedent knowledge (Kartashova \& Akhmedzianova, 2019).

Verbal and visual components of a meme can correlate as follows: they can be interdependent, i.e., the image depends on the text, without which the meaning of the image is lost or can be incorrectly decoded by the recipient, or they can be independent, i.e., the verbal commentary performs a complementary function. To accurately analyze and perceive memes, the viewer needs some certain knowledge (presuppositions), including extralinguistic (social knowledge, knowledge of science, culture, and history), logical (the idea of natural relations between events), and linguistic presuppositions (knowledge of language reality, set expressions, and graphic means important for the perception of implicit information). Deciphering the visual codes of memes requires an understanding of graphics (features of color, background, size, shape, etc.) and historical and cultural knowledge (information about the depicted object, person, event, or phenomenon). However, even if the recipient does have the required knowledge, it is not a guarantee of successful perception: the perception of denotative information does not pose a problem for the recipient, as it is based on general knowledge, while the decoding of the connotative code, which contains the information based on associative bonds, requires additional background knowledge. The most important element of social and cultural communication is the image created through metaphorics, semantics, associativity, and the principles of imaginative thinking. It seems necessary to take these specific features into account when studying Internet memes in the context of media discourse of such diametrically opposed cultures as Russian and Chinese (Davison, 2012; Shi-xu, 2014; Vartanova, 2019; Vartanova, Cherevko, Tolokonnikova \& Dunas, 2019; Yunhui, 2010; Dunas \& Vartanov, 2020).

Within this approach, it is beneficial to use a typology suggested by L. Shifman (2013) for the analysis of Internet memes, consisting of content (the idea and ideology a meme conveys), form (an aspect Shifman calls the "physical incarnation 
of the message", but which I will adjust to mean the memetic category of utility), and stance (which "depicts the ways in which addressers position themselves in relation to the text, the linguistic codes, the addressees, and other potential speakers") (Shifman, 2013, p. 367).

Researchers in the field of psychology note that such a global challenge as the COVID-19 pandemic has increased the risks for mental health: some affected individuals have been reported to exhibit stress, anxiety, depressive symptoms, fear, denial, and anger (Kang et al., 2020; Pestova \& Safonov, 2020). Semiotically, users employ the meme to convey dark humor representative of some aspect of modern society or a current event: "The boulder [which killed the Pompeii Victim, authors], with its lethal power, forces the victim, dead for over 2,000 years, to live again, memetically, only to be killed recursively in the force of the meme's function (also) as a joke" (Wiggins, 2019, p. 18).

Indeed, the level of stress that people all over the world have experienced and still experience in these new circumstances is significant, which cannot but lead to serious consequences such as the impact impairing individuals' immune systems (Tao, 2006), negative impacts on daily life and reduced well-being (Holbrook et al., 2005) and others. In particular, the researchers find it important to analyze the mental state of people from different age groups in such conditions, including students' mental health (Jieling et al., 2014), as well as potentially aggravating students' psychological distress during the COVID-19 outbreak (Xiao Zh. \& Benxian Y., 2020).

To understand the role of media as a tool that individuals can use to manage the stresses caused by events such as the COVID-19 pandemic, recent research showing the aspects of the problem has been useful (Smirnova et al., 2020; Fang \& Haochen, 2020; Xiao \& Benxian, 2020).

\section{MATERIALS AND METHODS}

The purpose of the study is to analyze and compare visual perceptions of Internet memes related to the COVID-19 pandemic by graphic arts experts, artists, arts and culture researchers, and young people (students) from Russia and China.

The study focuses on ethnocultural sensitivities in the visual perception of Internet memes related to the COVID-19 pandemic by representatives of Russian and Chinese cultures. The research involved two groups of participants: undergraduate and master students of faculty of psychology and faculty of arts (aged 18-23, $\mathrm{n}=108$ ) affiliated to Russian and Chinese universities accordingly, and university educated experts in visual arts and culture ( $n=12$, aged 25-65) affiliated to the faculties of arts and cultural studies at Russian and Chinese universities. The study design splits the process into two phases. The first involved 
the students, Chinese $(n=50)$ and Russian $(n=58)$, while the second involved the experts, Chinese $(n=6)$ and Russian $(n=6)$. COVID-19 related memes from Russia and China formed the material of the study. The survey in both rounds offered the participants a questionnaire containing multiple-choice questions and the possibility to add comments if needed.

Russian and Chinese memes used as the material for the study were selected randomly relying on the authors' subjective evaluation, judging which samples may be more semantically loaded and the interpretation of which might presuppose a solid cultural-specific background. Memes needed to meet criteria to be included in the study. They had to have a distinctive visual component that could be represented by any object (people, flora and fauna, symbols, etc.) and have any format, i.e., realistic and fantasy images, photographs, drawings, paintings. The memes were borrowed from traditional media (print press, tv broadcast) memes $(\mathrm{N}=400)$, and also digital (Internet) memes $(\mathrm{N}=267)$ were selected, which Chinese and Russian students ( 5 of each) collected between April 15 and May 15, 2020. Based on the selection criteria, the study chose 10 memes, 5 Chinese and 5 Russian that were the most illustrative.

To evaluate the selected memes, the study developed a perception test questionnaire, which was used in both phases of the study. Participants (both students and experts) were sent the questionnaire and the memes to their email addresses. In the first phase, the students had to indicate their liking using the scores from 3 to 0 (where 3 was high; 2 was medium; 1 was low; and 0 was neutral) for each of the 11 characteristics listed for each meme, which were as follows (displayed in italics throughout the text, as well as the additional comments provided by respondents): interesting - boring, relevant - outdated, important - useless, bright - obscure, cheerful - depressing, thought-provoking - not thought-provoking, smart and inventive - unoriginal, attractive - annoying, hinting - straightforward, meaningful - banal, kind - grim.

The students were also asked: to suggest a name for each meme, the most popular of which was selected; to describe the mood and associations evoked by the meme; as well as to rate each on a 5 -point numerical scale with ' 5 ' as the highest score. In addition, each respondent was asked in an open question to give a brief verbal description to each meme. In the second phase, the experts used the same perception test to positively or negatively evaluate the same 11 characteristics of each meme. In addition, the experts had to rate, on a scale from one to five, the visual features of each meme. The experts were also asked to provide a detailed description of those visual features, including the meme's visual idea, choice of composition and colors, general visual form of the meme, and the relationship between the visual and text images.

The hypothesis of the study is that the perception of image reality, which manifests as the relationship between the universal and the particular or the 
subjective and the objective, is fundamentally influenced by social and cultural aspects. Social, worldview, cultural, ethical, professional, and other contexts generate image realities that are unique for each social and cultural group. The interpretation of memes as a genre of image reality is based on the joint functioning of three interrelated elements: the visual imagery, the verbal component, and the socio-cultural image. Furthermore, precedence is the key component of memes, since the lack of background knowledge and the failure to understand the cultural code embedded in the meme prevent the "decoding" of semantics and, in fact, desemanticize the meme.

\section{RESULTS}

This section indicates the results of questionnaire responses of the participants (12 experts and 108 students from Russia and China) and conducts a comparative analysis of their evaluation of the 10 Internet memes related to the COVID-19 pandemic. Each meme has a Table which shows both the criteria for evaluating the memes on a scale from 1 to 5 and the average scores of the experts and students.

The paper will then present quantitative characteristics of the memes (see the figures in the corresponding tables) and qualitative assessments given by the respondents during the experiment.

Table 1. Evaluation of the Russian meme "TV Show"

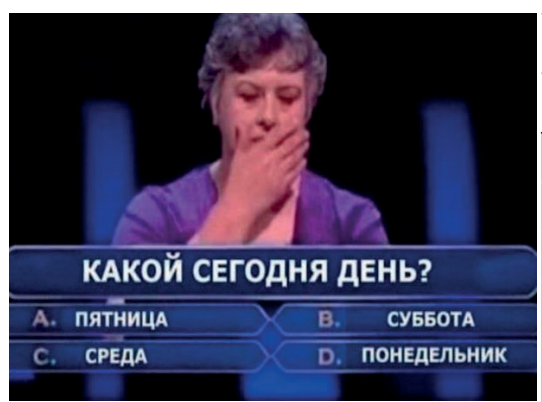

\begin{tabular}{|c|c|c|c|}
\hline \multicolumn{4}{|c|}{ Experts } \\
\hline & RU & $\mathrm{CH}$ & avg \\
\hline Visual idea of the meme & 2.8 & 2.3 & 2.5 \\
\hline Choice of composition & 3.3 & 2.3 & 3.8 \\
\hline Choice of colors & 3.3 & 2.5 & 3.0 \\
\hline $\begin{array}{l}\text { Visual form of the } \\
\text { meme in general }\end{array}$ & 3.1 & 2.8 & 3.0 \\
\hline $\begin{array}{l}\text { Relationship between visual } \\
\text { and text images of the meme }\end{array}$ & 4.1 & 3.1 & 3.6 \\
\hline $\begin{array}{l}\text { Overall score given by experts } \\
\text { (visual and text elements) }\end{array}$ & 3.0 & 2.7 & 2.8 \\
\hline \multicolumn{4}{|c|}{ Students } \\
\hline & RU & $\mathrm{CH}$ & avg \\
\hline $\begin{array}{l}\text { Overall score given by students } \\
\text { (visual and text elements) }\end{array}$ & 3.0 & 2.7 & 2.9 \\
\hline
\end{tabular}

Note: Russian language question and answers in English:

"What day is today?" A. Friday. B. Saturday. C. Wednesday. D. Monday 
The text in this meme is important [to speakers of Russian] because if they were erased the concept of the meme would lose its meaning. This meme is one of those visual images that mass audiences can easily decode because it is an actual scene from the TV show Who Wants to Be a Millionaire? The text and the body language of the woman contestant are also quite unoriginal and international. Indeed, the meme got almost the same scores from the Russian and Chinese students and experts, but the key qualitative characteristics were different.

Experts from Russia described the meme as banal, cliched, and stereotypical; the visual idea is not original. The opinion of the Chinese experts was divided: some participants did not understand the idea; others thought it was not of current interest, and yet others said the meme was interesting. The students had a better understanding of meme's meaning noting that it is important, relevant, creative, attractive, meaningful, kind, and cheerful. The experts gave a higher score to characteristics and gave additional comments of the memes evaluating them as thought-provoking and smart and inventive. The following paired categories were rated with the greatest difference: cheerful: depressing (a difference of 1.7 points), kind: grim (a difference of 1.6 points), and meaningful : banal (a difference of 1.5 points).

The Russian experts unanimously gave a higher score to the visual component across all categories: visual idea, choice of composition and colors, visual form, and the relationship between the visual and text images. The higher score given by the Russian experts may be because, for Russian viewers, memes based on footage from this TV show are familiar and have quite stable semiotics. As a rule, memes referring to a show or a film are a combination of two components, a question with a presupposition and answers that are either completely inappropriate or duplicate each other (verbal component) and a contestant with a serious, puzzled expression (visual component). The combination of a preposterous question with answers and a contestant deep in thought highlights the absurdity of the situation.

The respondents gave the highest score to the relationship between the visual and text images, since it is the combination of verbal and visual components that plays a crucial role in "decoding" semantics in this case. However, it is utterly predictable that the Russian speakers were able to better grasp how the elements are interrelated (the scores differ by as much as one point: 4.1 by the Russian experts vs. 3.1 by the Chinese experts). 
Table 2. Evaluation of the Russian meme "Cat"

\begin{tabular}{|c|c|c|c|c|}
\hline & \multicolumn{4}{|c}{ Experts } \\
\hline
\end{tabular}

Note: Translation from Russian: Come up and sit down: we need to talk. Igor, when are going to work

This meme is based on the anthropomorphism of the cat as the master of the house who allows people live on its territory but only under certain conditions. The image is well known on the Internet. The text and the image are interrelated and of equal value: the verbal component in this case supports the semantics of the visual image. It would be impossible to interpret the meme without background knowledge and similarly, the meme loses its semantic load if it is not supported by the text.

This meme was rated higher than the previous one by the Russian and Chinese respondents. The students and the experts gave it almost the same score: 3.4 vs. 3.6, respectively. Both respondent groups gave high scores to understandability, kindness, attractiveness, and relevance of the meme. Unlike the previous meme, this meme got a higher score in terms of creativity being rated 4.8 by the experts and 4.2 by the students. However, such criteria as importance, thought-provoking ability, and meaningfulness were rated as average.

While the visual form of the meme got approximately the same score from the Russian and Chinese experts (3.8 and 3.5 points, respectively), it is worth noting that the relationship between the visual and text images of the memes was rated by the Chinese experts higher than by the Russian experts (3.8 vs. 3.3).

The Russian experts gave quite a high score to the visual idea of the meme, whereas the Chinese experts did not find it interesting: the Russian experts gave higher scores to the choice of composition and colors (4 and 3.6, respectively). 
Interestingly, the Chinese experts in their free responses emphasize the facial expression of the cat and the preference for animal images in memes. As for the Russian experts, they claim that such stories are often used in memes and therefore lack originality; however, they consider such memes to be positive and appropriate.

Therefore, despite the general positive impressions of the meme and the positive emotions it evokes in all recipients, the hackneyed image of the cat has resulted in lower scores for the criteria of visuality.

Table 3. Evaluation of the Russian meme "Green"

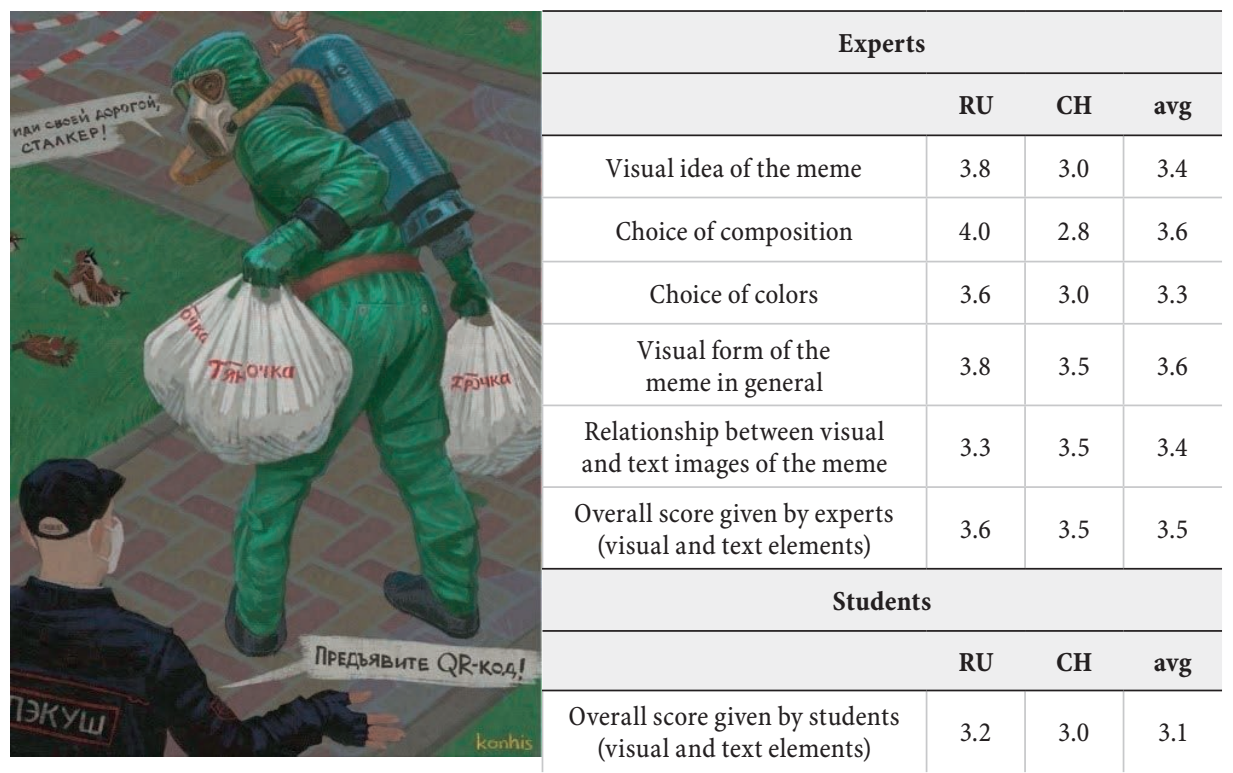

Note: Translation from Russian: Guard in black: Show me your QR-code. Man in Green: Go on your way, stalker

Unlike the previous two memes, "Green" belongs to a series of memes created by art illustrator M. Sidorenko (whose digital byline is konhis), which can be interpreted relying on the image solely, without considering can be deciphered the verbal component: the dialog accompanies the visual image, supplementing the message with universal background knowledge.

This meme got a higher overall score of 3.5 from the experts than from the students who rated it at 3.1, with the least difference between the Russian and Chinese respondents, which is due to the international nature of the meme's key images.

Remarkably, the Russian and Chinese participants interpreted the mood of the meme in diametrically opposite ways, although in both cases the quantitative indicators were close to neutral. The Russian respondents, both experts 
and students, tended to consider the meme rather grim (rated 2.6 by the experts and 2.9 by the students), whereas the Chinese respondents, by contrast, said that it was kind and bright (5.3 and 4.5, respectively). Moreover, the Chinese respondents found it more interesting (the average score is 4.9), relevant (5.6), and important (5.3). The Russian respondents gave higher scores for meaningful (5.1) and smart and inventive, the latter being much more highly rated by the students than the experts (5.8 vs. 4 ).

The visual idea of the meme received better recognition from the Russian experts. Their scores for the choice of composition (4), choice of colors (3.6), and visual form (3.8) were substantially higher than those by their Chinese colleagues. However, the relationship between the visual and text images of the meme was rated slightly higher by the Chinese experts (3.5) than the Russian ones; in their free responses, most Chinese experts stated that the story was interesting, funny, and self-explanatory and that it reflected the reality.

The Russian experts gave varied comments. Most of them believe that the meme has an original idea and is relevant; others failed to understand or appreciate it. Perhaps such a discrepancy, which is especially noticeable among the Russian experts, may stem from the instance that if the recipient has no knowledge of the "fans' universe", its characters and vocabulary, it will be difficult to decipher the connotative code of the meme.

Table 4. Evaluation of the Russian meme "Boys"

\begin{tabular}{|c|c|c|c|c|}
\hline & \multicolumn{4}{|c|}{ Experts } \\
\hline & Scores $(5$ to 1$)$ & RU & $\mathrm{CH}$ & avg \\
\hline & Visual idea of the meme & 4.6 & 4.3 & 4.5 \\
\hline & Choice of composition & 4.5 & 4.5 & 4.5 \\
\hline & Choice of colors & 4.5 & 4.2 & 4.4 \\
\hline & $\begin{array}{l}\text { Visual form of the } \\
\text { meme in general }\end{array}$ & 4.3 & 4.2 & 4.3 \\
\hline & $\begin{array}{l}\text { Relationship between visual } \\
\text { and text images of the meme }\end{array}$ & 4.1 & 4.2 & 4.1 \\
\hline москвичи мғнаит номер пома & Overall score given by experts & 4.5 & 4.0 & 4.2 \\
\hline \multirow{4}{*}{$\begin{array}{l}\text { МОСКВИЧИ МЕНЯЮТ НОМЕР ДОМА, } \\
\text { ЧТОБЫ ПОГУЛЯТЬ }\end{array}$} & (visual and text elements) & & & \\
\hline & \multicolumn{4}{|c|}{ Students } \\
\hline & & RU & $\mathrm{CH}$ & avg \\
\hline & $\begin{array}{l}\text { Overall score given by students } \\
\text { (visual and text elements) }\end{array}$ & 3.7 & 3.6 & 3.7 \\
\hline
\end{tabular}

Note: Translation from Russian: Moscow residents are changing the number of their apartment building to be allowed to go for a walk 
This is based on the reproduction of the painting by Fyodor Reshetnikov "For Peace!" (Za Mir!) (1950). The respondents gave it the highest score, even though the story is about the restrictions imposed in Moscow in summer 2020, when each apartment block required an official walking schedule. Yet, the survey demonstrated that the meaning of the meme was clear even to an international audience. It is interesting to note that the meme is effective only when the visual and verbal components are considered simultaneously: unlike the meme "Green" (Table 3), the inscription here performs a supportive sense-making, rather than an auxiliary, function.

The Russian and Chinese students were more critical in their evaluation (3.7 and 3.6, respectively) than the experts (the meme was rated 4.5 by the Russian experts and 4 by the Chinese experts). At the same time, the Russian students did not find the meme interesting, whereas the Chinese respondents (both students and experts) and the Russian experts rated this criterion quite highly (5.1 to 5.4). All the groups mentioned that the meme is attractive, creative, meaningful, and relevant. Nevertheless, the Chinese students and experts rated the thought-provoking ability of the meme twice as high as the Russians. Moreover, the Russian experts do not think that the meme was either relevant or important. The score for the mood criterion shows the greatest difference of one point: the students from both groups said that the meme made them feel better, whereas the experts' opinion was more neutral.

When evaluating the visual language of the memes, the experts from both countries were almost unanimous and gave high scores across all criteria. At the same time, the visual idea of the meme and the choice of colors seemed more expressive to the Russian experts (a rating of 4.6), whereas the relationship between the text and visual components got a slightly higher score from their Chinese colleagues. The respondents from both countries were unanimous in their high score (4.5) for the choice of composition. The choice of colors and the warm colors of the painting seemed more appropriate to the Russian experts than to their Chinese colleagues ( 4.5 vs. 4.2 ).

In their free responses, the Chinese experts gave positive feedback characterizing the color, style, and drawing technique as "good"; they also mentioned the expressive form. The opinion of the Russian experts was divided: some experts noted the interesting solution and originality, while others emphasized the propaganda message and the controversial use of socialist realism paintings as memes.

Therefore, the combination of the reproduction, which forms the basis of the meme and has artistic merits (elaborate form, composition, and choice of colors), and the original idea of the meme has had a positive impact on the scores among all groups of recipients. 
Table 5. Evaluation of the Russian meme "Hedgehog"

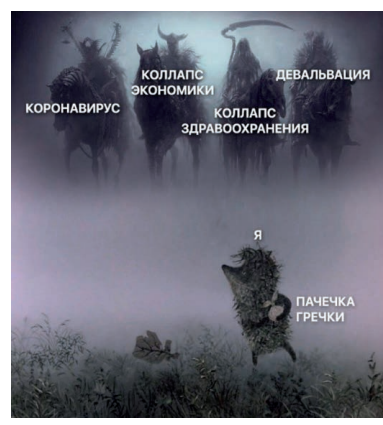

\begin{tabular}{|c|c|c|c|}
\hline \multicolumn{4}{|c|}{ Experts } \\
\hline & RU & CH & avg \\
\hline Visual idea of the meme & 3.6 & 3.8 & 3.7 \\
\hline Choice of composition & 3.5 & 3.6 & 3.5 \\
\hline Choice of colors & 4.0 & 3.2 & 3.5 \\
\hline $\begin{array}{l}\text { Visual form of the } \\
\text { meme in general }\end{array}$ & 3.5 & 3.6 & 3.5 \\
\hline $\begin{array}{l}\text { Relationship between visual } \\
\text { and text images of the meme }\end{array}$ & 3.5 & 3.3 & 3.4 \\
\hline $\begin{array}{l}\text { Overall score given by experts } \\
\text { (visual and text elements) }\end{array}$ & 3.6 & 3.3 & 3.5 \\
\hline \multicolumn{4}{|c|}{ Students } \\
\hline & RU & $\mathrm{CH}$ & avg \\
\hline $\begin{array}{l}\text { Overall score given by students } \\
\text { (visual and text elements) }\end{array}$ & 3.3 & 3.5 & 3.4 \\
\hline
\end{tabular}

Note: Translation from Russian: Upper row - Four Horsemen of the Apocalypse - left to right: Coronavirus, Economic Collapse, Health Care System Collapse, Devaluation; Lower single - I; a packet of buckwheat

This meme is based on Hedgehog in the Fog, a 1975 Soviet animated film directed by Yuri Norstein, with somewhat redefined images of the Four Horsemen of the Apocalypse.

Regarding the quantitative evaluation of the last Russian meme, the Russian and Chinese recipients shared almost the same opinion. The Russian experts rated it slightly higher (by 0.2 points) than their Chinese colleagues. However, the Chinese students liked the meme slightly better than the Russians (3.5 vs. 3.3).

The Chinese recipients gave higher scores across all categories: interesting, relevant, important, thought-provoking, creative, attractive, smart and inventive, meaningful, and kind. The Russian experts, who are familiar with the iconography and the etymology of the story, found the meme grim and gave it a low score of 2.1, whereas the Chinese experts thought it was quite bright and kind, giving it a score of 5.1 out of 7 , which is probably because the Chinese audience appreciates the use of animal images in memes.

When evaluating the visual idea, the respondents mentioned that the composition of the meme was not balanced: the respondents from both countries rated it 3.5. The Russian experts gave a higher score to the cold and obscure colors (4 vs. 3.2), which is in line with how they described the mood of the meme.

The textual commentary again performs a sense-making function in this meme and cannot be understood and decoded by the recipients. Therefore, deciphering the connotative information of the meme presented certain difficulties for part 
of the foreign audience. The relationship between the text and visual images was rated 3.5 by the Russian experts vs. 3.3 by the Chinese experts.

In their free responses, the Chinese experts pointed out that the forecast was realistic and the future was uncertain for everyone. The Russian experts were more critical: they mentioned that the meme is overloaded, unbalanced, and ill-considered. This evaluation most likely results from a combination of international components, i.e., the most pressing global challenges presented by the Four Horsemen, and a concept peculiar to an exceptional reality, i.e. a bag of buckwheat. The Chinese audience paid attention to the universal nature and importance of the challenges in the first place, whereas the Russian experts focused on the local concept, which is considered by the Russian-speaking Internet users to be "unoriginal" and "primitive."

Table 6. Evaluation of the Chinese meme "Girls"

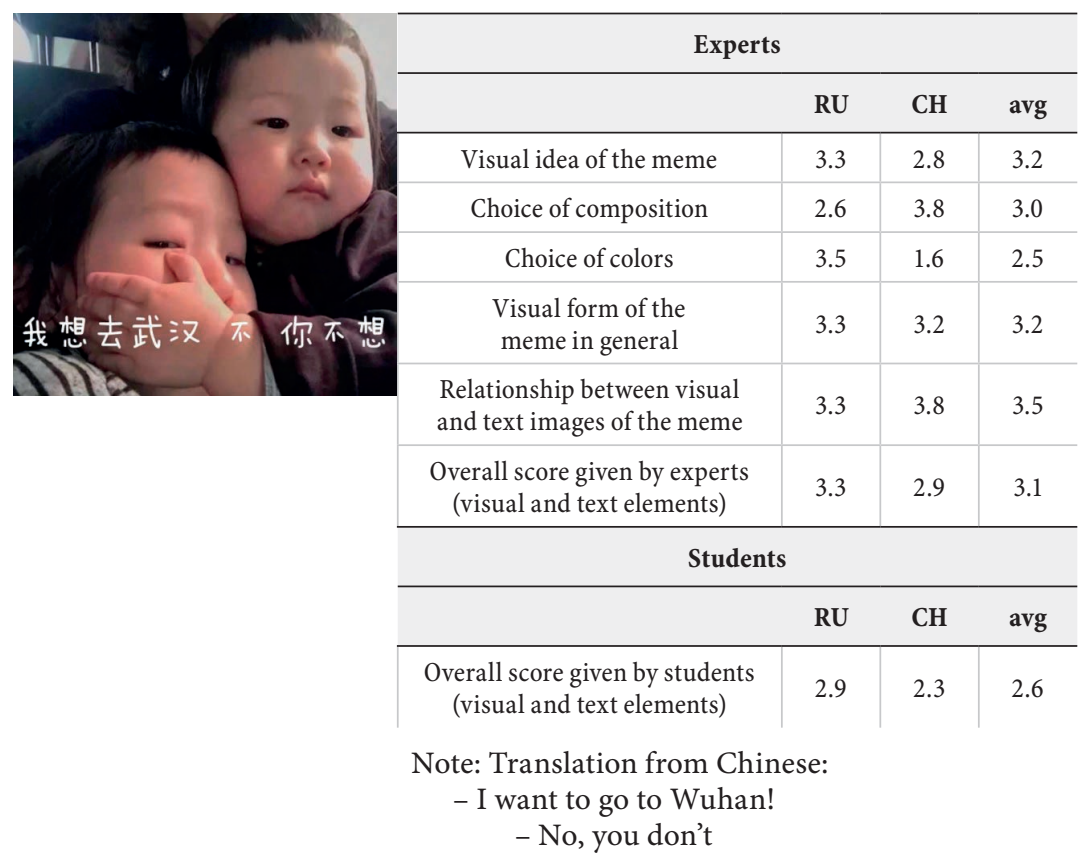

The story behind the photo used in this meme has a universal nature and may be interpreted in a variety of ways, depending on the text that complements the photo, as manifested by numerous times to this image was used to create memes on various subjects in China's Internet. Thus, the visual component of the meme in question cannot be interpreted on its own, without the verbal component. However, both the experts and the students mentioned that the semantics of the meme is clear. Besides, deciphering the semantic code of the 
meme does not require any culture-specific background knowledge, since the viewer relies on the body language (cf. TV Show).

This Chinese meme was rated low by all respondents. The Russian recipients found it more boring than the Chinese recipients (an average of 3.4 vs. 5). Perhaps Russian participants lacked the background knowledge that before the pandemic, Wuhan was known as a favorite destination for domestic and international tourism. For this reason, the Russian respondents to the survey considered it rather useless (rated 3.1 by the experts and 2.8 by the students); depressing (rated 2.5 by the experts); lacking thought-provoking ability (rated 2.7 by the students); unoriginal (3.4); too straightforward (3), rather annoying (3.5), and quite grim (rated 2.5 by the experts).

The experts gave an overall higher score to the meme than the students. The greatest difference in scores given by two categories of the respondents was 0.7 for the criterion of relevance: the experts found the meme more relevant than the students.

The visual form of the meme received almost the same scores averaging 3.2 , whereas the visual idea received a higher score from the Russian experts (3.3 vs. 2.8). The most diverging scores were given to the choice of colors for the picture ( 3.5 by the Russian experts and 1.6 by the Chinese experts). The Chinese experts thought that the choice of composition was more balanced (3.8), as was the case with the relationship between the visual and text components (3.8).

When responding to the open-ended question, the Chinese experts described the meme as usual, unoriginal, and banal. The Russian recipients described it as uninteresting, sad, uninspiring, and pessimistic. Thus, the translated meme can be understood by the audience who speaks another language, but it cannot trigger any strong emotions and it is generally rated quite low.

The difference in the perception of the meme by the Russian and Chinese respondents may be due to different media contexts in China and Russia. Lower scores by the Chinese respondents might be affected by the allegations against China and Wuhan on spreading COVID-19 made by the global media, which intensified during this period. 
Table 7. Evaluation of the Chinese meme "Dog"

\section{不出门 \\ 不添乱 \\ 我为国家做贡献}

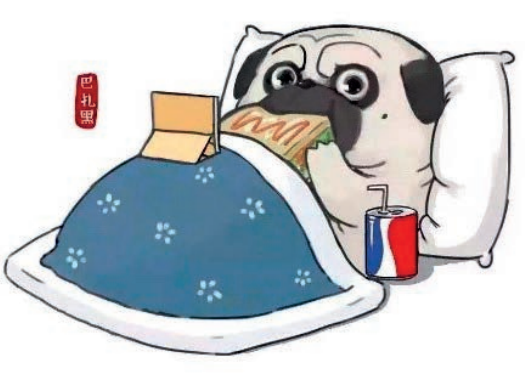

Note: Translation from Chinese: I am staying at home! I am not in a panic! This is what I do for my country

This meme continues the trend of using animals as the main characters of memes to convey meanings allegorically and impersonally. The symbols of staying at home and having rest that are easily understandable in many cultures, such as a soft pillow, a cozy blanket, a tablet, a soda can, and a sandwich, complement the text message of the meme. Stylistically, the picture is close to a comic strip, which also broadens the potential audience and render it suitable international viewing. The author of the meme uses signs that are understood by most of the recipients, which should resonate with the general public.

Unlike the students, the experts gave a higher score to this meme almost across all categories. The score for such a criterion as smart and inventive shows the greatest difference ( 4.5 by the experts and 3.7 by the students). There is a great difference between the scores given by the Chinese and Russian experts: the Chinese colleagues gave higher scores (+value) in all categories, including creative $(+2.2)$, thought-provoking $(+2)$, attractive $(+2)$, interesting $(+1.7)$, smart and inventive (+1.7), kind (+1.6) appropriate and important (+1.5), and meaningful (+1.5).

When evaluating the visual components of the meme, the experts from both countries were more unanimous and gave them a score from 3.2 to 4.3. The scores for the relationship between the visual and text components demonstrated the greatest difference of 0.8 points, with the Chinese experts rating it higher. In addition, some Russian experts pointed out that they were not able to fully discover the connection between the verbal and visual components. In their free responses, they made the following comments: visually understandable; banal; 
joke not clear; unoriginal. The Chinese experts described the meme as interesting, true to life, and relevant.

The findings show that in this case the Russian recipients lacked the sociocultural background knowledge to fully decipher the semiotic hybrid of the meme, which affected the level of their scores and the characteristics given to the meme.

Table 8. Evaluation of the Chinese meme "Lunch"

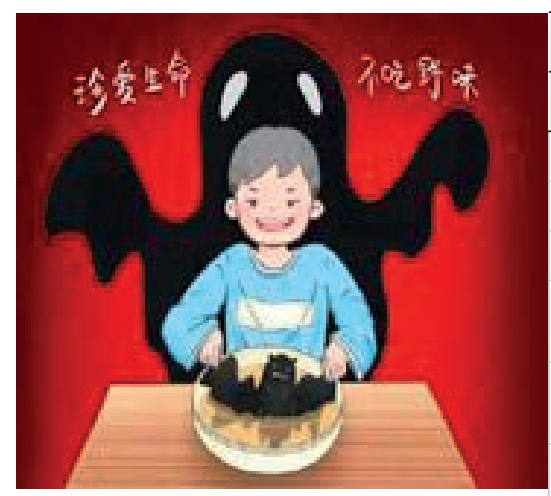

\begin{tabular}{|c|c|c|c|}
\hline \multicolumn{4}{|c|}{ Experts } \\
\hline & RU & $\mathrm{CH}$ & avg \\
\hline Visual idea of the meme & 3.1 & 2.3 & 2.7 \\
\hline Choice of composition & 3.8 & 2.2 & 3.0 \\
\hline Choice of colors & 3.8 & 2.3 & 3.0 \\
\hline $\begin{array}{l}\text { Visual form of the } \\
\text { meme in general }\end{array}$ & 3.5 & 2.2 & 2.8 \\
\hline $\begin{array}{l}\text { Relationship between visual } \\
\text { and text images of the meme }\end{array}$ & 3.5 & 2.3 & 2.9 \\
\hline $\begin{array}{l}\text { Overall score given by experts } \\
\text { (visual and text elements) }\end{array}$ & 3.1 & 2.2 & 2.6 \\
\hline \multicolumn{4}{|c|}{ Students } \\
\hline & RU & $\mathbf{C H}$ & avg \\
\hline $\begin{array}{l}\text { Overall score given by students } \\
\text { (visual and text elements) }\end{array}$ & 2.2 & 2.9 & 2.6 \\
\hline
\end{tabular}

Note: Translation from Chinese: Take care! Don't eat it

This meme got the highest score of 3.1 from the Russian experts and a much lower score of 2.2 from the Chinese experts and the Russian students. The scores vary widely by category. For example, the Russian students gave a score of 6.1 for interesting opposed to the Chinese students who gave a score of 4 and the Chinese experts with only 2.3 (maximum difference of 3.8 points). The Russian students gave a score of 6.6 for cheerful opposed to 1.6 given by the Russian experts (a five-point difference). The Chinese recipients are confident that the meme is thought-provoking but the Russian recipients tend to disagree. The Chinese respondents also gave substantially higher scores to categories such as important, creative, attractive, smart and inventive, meaningful, and kind.

The analysis of the scores given to the visual component of the meme showed different results: the picture is based on an original story, has a unique style, and is not banal. The composition uses the method of multiplication of familiar bat contours and simultaneous scaling of objects. The Russian experts gave higher scores than their Chinese colleagues to the visual idea of the meme $(+0.8)$; the choice of composition $(+1.6)$ and colors $(+1.5)$; the visual form $(+1.3)$, and the relationship between the visual and text components $(+0.9)$. The text is a conventional poster slogan 
which intensifies and adds to the image. Therefore, this meme is interpreted because of the verbal and visual components, as well as the need to know what happened.

It is, however, possible that this meme has lost some of its relevance compared to the first months of the pandemic, after Chinese scientists disproved the theory that the coronavirus passed to humans from bats, and thus the background knowledge became obsolete. In this regard, the groups of experts gave mostly negative evaluations in their free responses. Key words by the Chinese experts were: boring; not true; not attractive; looks like a textbook illustration; gives a lecture. Key words by the Russian experts were: not funny; specific humor; strange presentation; depressing; incomprehensible; grim; gives an ultimatum.

The analysis of the findings showed that the Chinese respondents reacted extremely negatively to the idea of this meme, which alluded to the exotic culinary traditions of the Celestial Empire. Amid the allegations of Chinese being unscrupulous eaters that poured from the global media during the COVID-19 pandemic, this meme provoked, quite logically, a rejection from the Chinese recipients, hence low scores.

Table 9. Evaluation of the Chinese meme "Sunset"

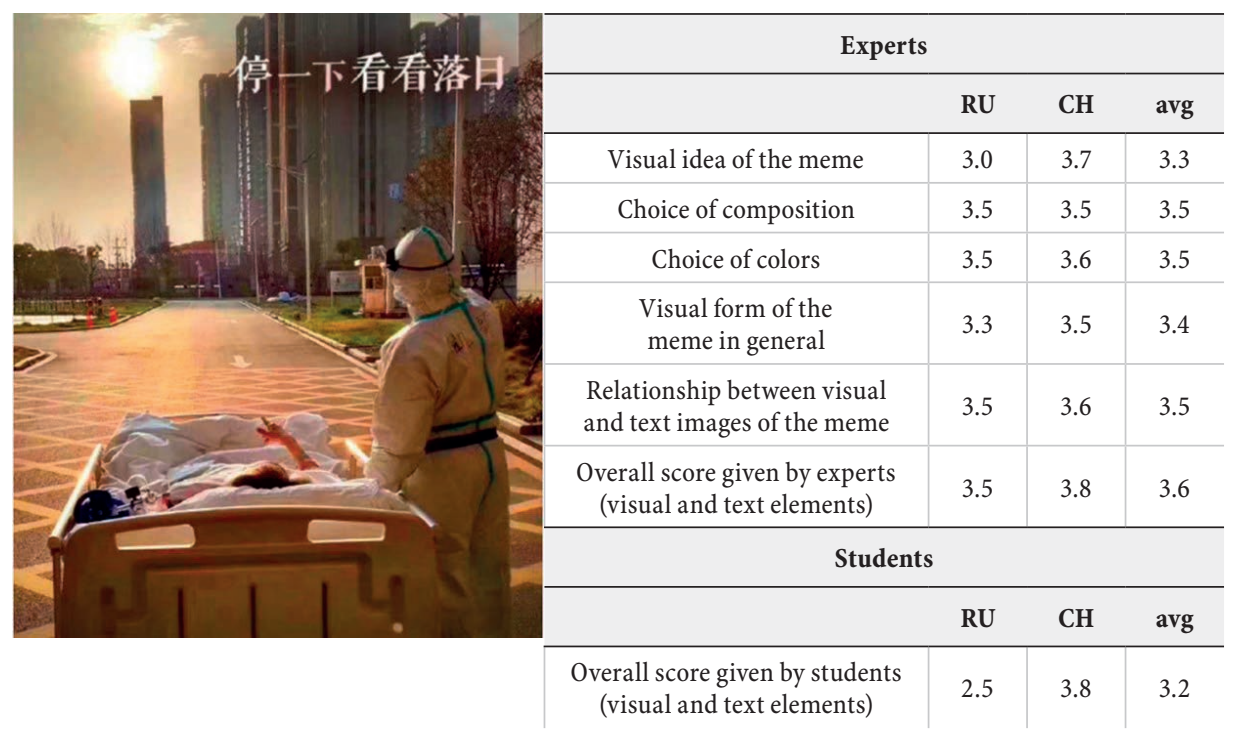

Note: Translation from Chinese: Stop and watch the sunset

This meme illustrates the need to interpret three key components: visual, verbal, and conceptual. This meme got higher scores from the Chinese respondents, who were aware of the photo appearing in the media of a patient being transported to another hospital building admiring the sunset. The Russian experts did not understand the meaning of the meme ( 3.5 out of 7 ), unlike the Chinese experts who understood it perfectly ( 6.6 out of 7, i.e., a difference 
of 3.1 points). The Russian experts found the meme rather annoying (3.5) and grim (3.3), whereas the Chinese experts found it rather attractive (5.5) and kind (4.8). The experts gave overall higher scores than the students for categories such as important, clear, thought-provoking, attractive, and kind; at the same time, the students found the meme quite creative.

The scores given to the visual idea of the meme were close, with a difference of max. 0.7 points. The overall impression of the Chinese experts is hope, positive energy, human love, and a positive meaning; whereas that of the Russian experts is philosophical nature, an unoriginal concept, and an unclear idea.

When evaluating the visual component of the meme, the Chinese experts were also more tolerant than their Russian colleagues. The message of the meme refers to contemplation as the most important category of the Chinese culture and philosophy. Yet, the allegory of the setting sun can also carry a negative connotation: the sunset of a person's life in this world, which was reflected in the scores of the Russian recipients. So, the attitude towards the meme is ambivalent among an ethnically different audience which is not familiar with the event that was the source of the meme.

Table 10. Evaluation of the Chinese meme "Piggy"

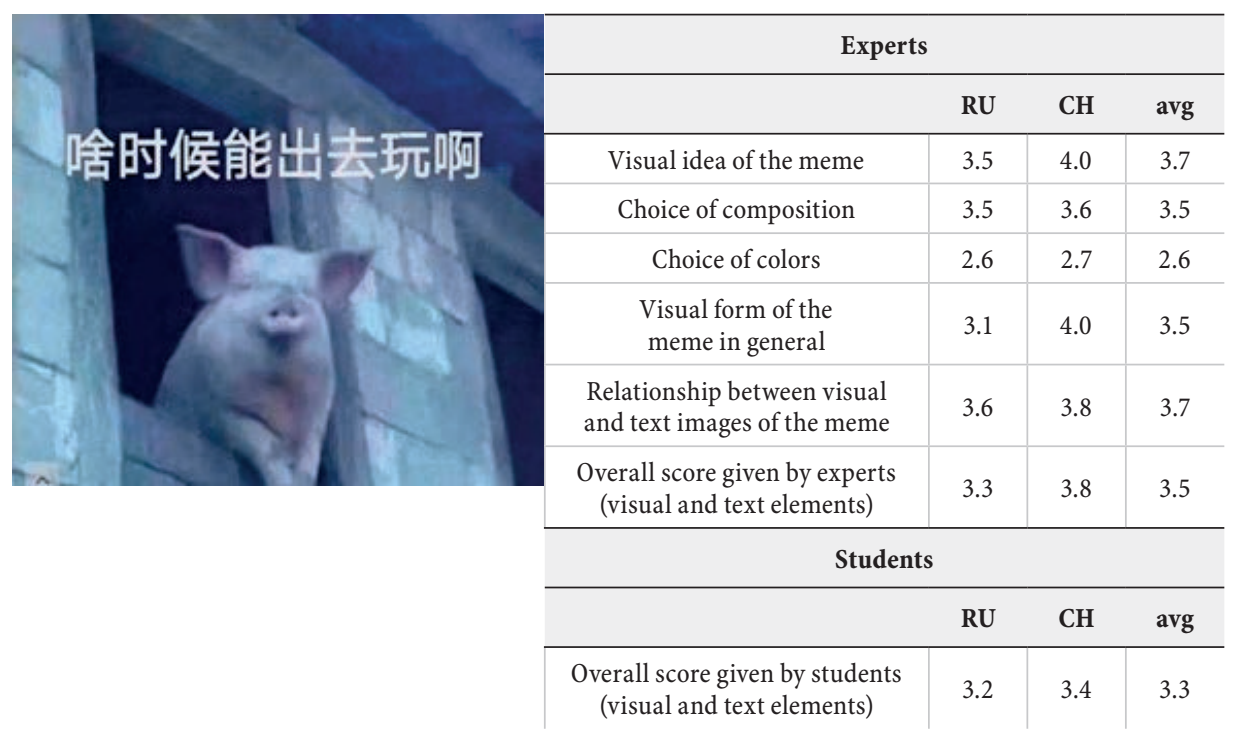

Note: Translation from Chinese: When will I be allowed to go out for a walk

The message of this meme is simple and clear; the translated meme can be easily understood by the general public. To interpret this sample, one does not require culture-specific background knowledge: it is sufficient to "grasp the moment," international in nature, which is confirmed by the overall high scores for this criterion (6.5 out of 7). 
However, the Chinese recipients gave much higher scores to this meme and found it interesting (6.3), relevant (6.3), appropriate and important (5.8), cheerful (5.8), thought-provoking (5.1), creative (5.1), attractive (6.3), smart and inventive (5.5), meaningful (5.6), and kind (5.6). The Russian recipients were not impressed by the meme; the gap in their scores reached 3.0 points.

A similar trend can be observed in the analysis of visual characteristics of the memes. The Chinese experts gave higher scores across all categories, although the difference was not that great and did not exceed 0.9 points for the visual form of the meme. Similar to the Russian meme "Boys", the experts from both countries gave the highest scores to the relationship between the visual and text components (3.6 and 3.8) and the visual idea of the meme. The composition of the meme is comparable to the Russian meme "Cat" and presents an animal communicating the ideas of a large portion of the society; its scores are also close (3.5 and 3.6).

The choice of colors (cold monochromatic colors) got the lowest scores: 2.7 and 2.6 by the Chinese and Russian experts, respectively. Nevertheless, despite the minimal difference in the quantitative parameters, the description by the Chinese experts is more positive. The Chinese experts described the visual idea as very nice; a good and reasonable combination; interesting; this is life. At the same time, the Russian experts said that the idea was good but poorly implemented; sad; uninspiring; banal; amusing but not profound.

Therefore, even though the idea of the meme was clear to the participants of both groups, the meme evoked more positive emotions from the Chinese recipients. This is partly because the main character of the meme, the piglet, is quite revered in the Chinese culture and astrology, whereas in the Russian tradition this animal carries rather negative connotations.

The analysis of the summary results of the expert survey showed that in 18 cases the scores of Chinese experts were higher (the Chinese memes in 12 cases with a difference of 1.2 points, the Russian memes in 6 cases with a maximum difference of 0.5 points). In two cases, the experts from both countries gave the same scores: the experts were unanimous in their evaluation of the choice of composition for the memes "Boys" (4.5) and "Sunset" (3.5). As to the other 30 scores, the Russian experts were more favorably disposed than their Chinese colleagues and gave higher scores to both Russian and Chinese memes. From our point of view, the cause lies in a combination of factors, with the key ones being the difference in the types of knowledge - background, linguistic, extralinguistic, historical and cultural, and graphic. The last is undoubtedly influenced by the differences in the approach to professional art education provided in Russia and China.

The Chinese experts were generally more critical than the Russian experts across all categories of meme visuality: to the visual idea, the choice of composition and colors, and the visual form in general. The choice of colors was rated lowest ( 3.0 by the Chinese experts and 3.6 by the Russian experts). The visual form got 
3.4 from the Chinese experts and 3.5 from the Russian experts. The visual idea was rated 3.3 by the Chinese experts and 3.5 by the Russian experts. The choice of composition in general was rated 3.3 by the Chinese experts and 3.7 by the Russian experts. However, the general score for the relationship between visual and text images was the same for the Russian and Chinese experts, although the Chinese experts gave higher scores to 7 out of 10 memes. According to this criterion, the outsiders were the memes "TV Show", "Hedgehog", and "Lunch", which prompted controversial comments in the free responses. The important aspect in the evaluation of this category was that the information was transferred through two channels, verbal and visual. Moreover, their ratio may vary. In some cases, the image may provide more valuable information for understanding than the text, and vice versa. But in most cases they cannot exist separately from each other without losing their semiotic significance.

Regarding the difference in the feedback provided by the students and experts, it is to be noted that experts gave higher average scores to the relationship between the visual and text images ( 3.6 out of 5). The choice of colors was rated lowest (3.2); in this respect, the Chinese experts were especially critical (3.0 by the Chinese experts vs. 3.6 by the Russian experts). This pattern is observed in the evaluation of almost all memes, except for the monochromatic "Sunset" and "Piggy": the latter got a score of 4.0 from the Chinese experts for the visual form. Comparison of the visualization scores given by the experts and the students showed that they are generally very close (3.4 vs. 3.2), with the experts' scores just 0.2 points higher. These differences are also due to a more balanced, (both professional and personal) experience-based perception by the experts and a more spontaneous, emotional and critical perception by the young audience.

Moreover, there was a difference in the scores given by the experts and the students from different countries. Noticeably, both experts and students gave higher scores to those memes, including their visual component, that belong to their native language culture; this was particularly true in the evaluation of the Chinese memes, which got an average score of 3.4 from the Russian experts and 3.9 from the Chinese experts. Accordingly, the Chinese students gave a score of 3.3 to their country's memes, while the Russian students gave the Chinese memes a score of 2.7. The same holds true for the evaluation of the Russian memes, but the difference between the scores was more subtle ( 0.3 points for the experts and 0.1 points for the students). Therefore, it is fair to say that, in this case, the age-related and professional contexts had less influence on the evaluations of the memes than the nationality of the recipients. The perception of information based on general knowledge did not cause difficulties for any of the recipients, but decoding the connotative code, the information in which is based on various associative bonds, demonstrated a lack of knowledge of the culture, specific national features, and social realities of another country. 


\section{DISCUSSION}

The COVID-19 memes in such ethnoculturally different audiences as Russians and the Chinese perform a compensatory function for young people: the relevant content and a creative form of memes help students to overcome the hardships of the pandemic. A new communication medium, which offers entirely different information opportunities and relations between the audience and the medium, requires researchers to explore and understand these new realities, which affect, among other things, the functioning and audience perception of the content related to healthy living and the issue of the COVID-19 pandemic.

Further research may include a t-test for a broader group of respondents, and the study on how memes are perceived by respondents with according to their demographic profiles (gender, age, education, etc.), as well as the dispersion analysis of repeated-measures ANOVAs to examine differences in emotional responses to a range of memes. Another important line of further research would be the analysis of memes in the context of language cultures, and the study of the perception of memes in terms of content comprehensibility, the degree of involvement in the situation, and the level at which the cultural background may be needed to appreciate the meme. In addition, the immediate task of this project was to analyze the visual imagery of memes and its semiotic relationship to the text in greater detail.

It is suggested to conduct a more detailed study of the empirical data with reference to scientific research on the perception of visual aspects of communication, including memes. The paper mostly analyzes the findings of the current empirical study. However, it is essential to go deeper into the psychology of shape and color perception (Arnheim, 2004; Itten, 2020), etc. by ethnic groups (in this case, Russians and Chinese) in correlation with the empirical data we obtained. As the scope of this paper is limited, this should be the subject of further publications. Also in this respect, it would be beneficial to rely on the triple typology of memes proposed by Shifman (Shifman, 2014a) and refer to poststructuralist concepts (Barthes, 1977; Kristeva, 1980) to study the phenomenon of meme inter-iconicity. Another issue that requires discussion and further research concerns the deeper reasons (such as the influence of the media context, the psychology of perception) that can help explain both the gap in opinions between the Russian and Chinese recipients and, in some cases, their closeness. For example, "Sunset" was perceived differently but not, as Wiggins (2019) supposes, is it so important for us to know the specific reasons why the differences are perceived, as to why it is generally understood. 


\section{LIMITATIONS}

This research has four limitations. The first three concern the size $(\mathrm{N}=120)$ and the F:M gender ratio (X:Y) and geographical bias (predominantly Moscow) of the research sample. The fourth concerns the narrow scope of the linguistic cultures.

\section{CONCLUSION}

The analysis of the scores for meme visualization allows us to conclude that the interpretation of memes requires the analysis of three components: visual, verbal and background knowledge. Meme interpretation involves both culture-specific elements that can be read only by native speakers of a given culture and international phenomena, understandable to most people, regardless of their cultural and social background. When interpreting foreign memes, mainly international background knowledge and knowledge relevant at this point of time are captured. Moreover, culture-specific aspects in memes appear quite often as not to be "read" by representatives of another culture. If the conceptual component plays a leading role, the group of another national identity feels a lack of culture-specific background knowledge and considers such a meme as incomprehensible or uninteresting. In the process of meme perception, the evaluation by recipients is based on deciphering visual, cultural, ideological, linguistic, and semiotic codes. Linguistically and visually close structures are most successfully decoded, whereas memes belonging to some another culture are least successfully decoded. Overall, the analysis suggests that it is the semiotic hybrid that makes the meaning in the perception of native-language memes, whereas the visual imagery becomes dominant in the perception of foreign-language memes by both experts and students. This supports the idea the notion that "Internet memes are not merely content items and thus simply replicators of culture but are rather visual arguments, which are semiotically constructed with intertextual references to reflect an ideological practice" (Wiggins, 2019, p. 24).

As for memes evaluation by groups with an entirely different culture specific background, the differences highlighted in the research are related either to the degree of comprehensibility of the content, to the involvement in the situation, or to the knowledge of precedent facts. It is our understanding that this phenomenon is rooted primarily in the differences between the visual cultures of the countries. The cultural paradigms of the world are radically different for those who have European or Asian mentalities. The Russian cultural paradigm is more diverse and flexible, subject to change and development than the Chinese one, which is based on a commitment to strong traditions. This statement can also apply to the visual culture of China, where traditional painting 
has remained almost unchanged over the centuries. At the same time, the artistic traditions in Russia have changed dramatically and repeatedly, which could not but affect the way the perception of visual images is formed. If we extend this point further, we can say that traditional Chinese art is based on the graphic line. This is most likely why the Chinese recipients are more prone to complete a schematic image in their imagination, giving it new meanings. After all, this is inherent in the tradition of Chinese visual art, with its means of expression always being laconic, and images always being schematic. In Chinese painting, the message prevails over formal similarity, in which the clarity and precision of the artistic idea is valued; here we should recall the supremacy of the message over the form according to Shifman's typology (2014a). The attitude to color in images is also based on the color dogmas of Confucian and Taoist philosophy. The five elements (wood, metal, fire, water, and earth) are the source of the origin of China's traditional color culture. These elements have had a profound impact on Chinese society and have merged into the traditional colors of Chinese paintings; five colors (white, black, red, yellow, and green-blue) were selected to correspond to the concept of the five elements. Moreover, it is the presence of these colors that affects the evaluation of the color scheme. Therefore, we can argue that philosophical and aesthetic views inherent in the Chinese culture have a profound impact on the formation of the aesthetic perception of memes by Chinese recipients.

By contrast, the Russian painting tradition is characterized by the depth of space and three-dimensional images, the variety of subtle nuances, the fullness and depth of color combinations, and the realistic portrayal of reality. Moreover, the form in the context of Russian painting is no less important than the message. The psycho-emotional attitude to colors is also different and sometimes even opposing (for example, in the Chinese culture white is the color of emptiness, wisdom and mourning, whereas in Russia white is the symbol of purity and youth). It might therefore be said that the visual traditions of Russia based, among other things, on the principles of realist painting, with its precise and naturalistic manner of conveying forms, volume, and color combinations, could not help but influence the formation of sensitivities in the perception of memes by the Russian audience.

In conclusion, the social, philosophical and cultural context plays a fundamental role in the perception and evaluation of Internet memes, which, in turn, is determined by the reality paradigm predominant in a certain society at a certain time.

\section{DISCLOSURE STATEMENT}

The authors do not have any conflicts of interest. 


\section{ACKNOWLEDGEMENTS}

This research has been supported by the Interdisciplinary Scientific and Educational School of Moscow University "Preservation of the World Cultural and Historical Heritage".

\section{REFERENCES}

Aiello, G., \& Parry, K. (2019). Visual Communication: Understanding Images in Media Culture. SAGE Publications.

Arnheim, R. (2004). Art and visual perception. University of California Press.

Barthes, R. (1977). Image-music-text. Fontana.

Batibo, H. M. (2009). The inter-cultural dimension in the use of languages of wider communication by minority language speakers. Journal of Multicultural Discourses, 4(2), 89-102.

Blackmore, S. (1999). The Meme Machine. Oxford University Press.

Brodie, R. (1996). Virus of the Mind: The New Science of the Meme. Integral Press.

Castaño, D. (2013). Defining and Characterizing the Concept of Internet Meme. Revista CES Psicología, 6(2), 82-104. DOI 10.1177/2167479515584045.

Chaoqun, X., \& Ziran, H. (2007). 谢朝群, 何自然. 语言模因略说. 现代外语 [A Brief Introduction to Language Memetics]. Modern Foreign Languages, 1, 30-39. 0.3969/j.issn.1003-6105.2007.01.004.

Davison, P. (2012). The Social Media Reader. New York University Press.

Dunas, D., \& Vartanov, S. (2020). Emerging digital media culture in Russia: modeling the media consumption of Generation Z. Journal of Multicultural Discourses, 2, 186-203. DOI 10.1080/17447143.2020.1751648. Fang, Zh., \& Haochen, G. (2020). 张放, 浩辰.疫情心理时空距离对公众情绪的影响研究——基于新冠 肺炎疫期微博文本面板数据的计算分析[J].新闻界 [On the Influence of Temporal and Spatial Distance from Epidemic on Public Sentiment: A Computational Analysis Based on Panel Data of Web Text about COVID-19]. Journalism and Mass Communication Monthly, 6, 39-49. DOI 10.15897/j. cnki.cn51-1046/g2.20200514.017.

Holbrook, T.L., Hoyt, D.B., Coimbra, R., Potenza, B., Sise, M., \& Anderson, J. P. (2005). High rates of acute stress disorder impact quality-of-life outcomes in injured adolescents: mechanism and gender predict acute stress disorder risk. Journal of Traumatic Stress, 59, 1126-1130. DOI 10.1097/01. ta.0000196433.61423.f2.

Iloh, K. (2021). Do it for the culture: The case for memes in qualitative research. International Journal of Qualitative Methods 20. DOI 10.1177/16094069211025896.

Itten, I. (2020). Iskusstvo cveta [The art of color], Aronov.

Jieling, Ch., Xinchun, W., Panpan, Z., Xiao, Z., \& Guoyu, X. (2014). The relationship between PTSD and PTG: evidence from longitudinal study of teachers survived in Wenchuan earthquake. Psychological Development \& Education, 30, 75-81. DOI 10.16187/j.cnki.issn1001-4918.2014.01.010.

Kang, L., Li, Y., Hu, S., Chen, M., Yang, C., Yang, B., \& Liu, Z. (2020). The mental health of medical workers in Wuhan, China dealing with the 2019 novel coronavirus. The Lancet Psychiatry, 7(3), e14. DOI 10.1016/S2215-0366(20)30047-X. 
Kartashova, E., \& Akhmedzianova, A. (2019). Internet-mem kak osnovnoy vid kreolizovannogo teksta v samoprezentatsii «cheloveka govoryashchego» [Internet-meme as the basic type of creolized texts in self-presentation of «creative human»]. Vestnik Mariiskogo universiteta [Mari State University Bulletin], 3(35), 426-430. DOI 10.30914/2072-6783-2019-13-3-426-430.

Kress, G., \& van Leeuwen, T. (2020). Reading Images. The Grammar of Visual Design. (3rd Edition). Routledge.

Kristeva, J. (1980). Word, Dialogue, and Novel. Desire in Language: A semiotic Approach to Literature and Art. Columbia University Press.

Lobodanov, A. (2013). Semiotika iskusstva [Semiotics of Arts]. Izdatel'stvo Moskovskogo universiteta. Manovich, L. (2017). Theories of Software Culture. Krasnaya lastochka.

Manovich, L. (2020). Cultural Analytics. MIT Press.

Pestova, M., \& Safonov, E. (2020). Pandemiya novogo desyatiletiya: osveshchenie temy koronavirusa v SMI. Mediasreda, 1, 166-172. DOI: 10.24411/2070-0717-2020-10136.

Shi-xu. (2009). Reconstructing Eastern paradigms of discourse studies. Journal of Multicultural Discourses, 4(1), 29-48. DOI 10.1080/17447140802651637.

Shi-xu. (2014). Chinese Discourse Studies. Palgrave Macmillan.

Shi-xu. (2016). Cultural Discourse Studies through the Journal of Multicultural Discourses: 10 years on. Journal of Multicultural Discourses, 11(1), 1-8, DOI 10.1080/17447143.2016.1150936.

Shifman, L. (2013). Memes in a digital world: Reconciling with a conceptual troublemaker. Journal of Computer-Mediated Communication, 18, 362-377.

Shifman, L. (2014a). Memes in digital culture. MIT Press.

Shifman, L. (2014b). The cultural logic of photo-based meme genres. Journal of Visual Culture 3(13). DOI 10.1177/1470412914546577.

Shomova, S. (2018). Memy kak oni est' [Meme as they are]. Moscow: Aspekt Press.

Smirnova, O., Denissova, G., Svitich, L., Lin, Ch., Steblovskaia, S. (2020). Psychological and Ethnocultural Sensitivities in the Perception of COVID-19 Memes by Young People in Russia and China. Psychology in Russia: State of the Art, 13(4), 148-167. DOI 10.11621/pir.2020.0410.

Sorokin, Ju., \& Tarasov, E. (1990). Kreolizovannye teksty i ikh kommunikativnaya funkciya [Created Texts and their Communicative Function]. Azbukovnik.

Tao, J.H. (2006). Influence of acute psychological stress and exercise stress on the changes of immune function. Chinese Journal of Clinical Rehabilitation, 10, 126-128.

Vartanova, E. (2019). Russian media change as a cultural process. European Journal of Communication, 34(2), 205-210. DOI 10.1177/0267323119838088.

Vartanova, E., \& Gladkova, A. (2020). Old and new discourses in Emerging States: communication challenges of the digital age. Journal of Multicultural Discourses, 15(2), 119-125. DOI 10.1080/17447143.2020.1780244.

Vartanova, E., Cherevko, T., Tolokonnikova, A., \& Dunas, D. (2019). Changing patterns of digital news consumption among Russian journalism students. World of Media. Journal of Russian Media and Journalism Studies, 1, 7-31. DOI 10.30547/worldofmedia.1.2019.1. 
Vartanova, E., Gladkova, A., Lapin, D., Samorodova, E., \& Vikhrova, O. (2021). Theorizing Russian model of the digital divide. World of Media. Journal of Russian Media and Journalism Studies, 1, 540. DOI: $10.30547 /$ worldofmedia.1.2021.1

Wiggins, B.E. (2019). The discursive power of memes in digital culture: ideology, semiotics, and intertextuality. New York: Routledge.

Wiggins, B.E., \& Bowers, G.B. (2015). Memes as Genre: A Structural Analysis of the Memescape. New Media \& Society, 17(11), 1886-1906. 10.1177/1461444814535194.

Xiao, Zh., \& Benxian, Y. (2020). Social support and acute stress symptoms (ASSs) during the COVID-19 outbreak: deciphering the roles of psychological needs and sense of control. European Journal of Psychotraumatology, 11(1), 1-8. DOI 10.1080/20008198.2020.1779494.

Yunhui, Zh. (2010). [Research on Internet Language Grammar and Pragmatics]. Xuelin Publishing House. 